\title{
The star formation history in 12 SMC fields
}

\author{
Noelia E. D. Noël ${ }^{1}$, Antonio Aparicio ${ }^{1}$, Carme Gallart ${ }^{1}$, \\ Sebastián L. Hidalgo ${ }^{2}$, Edgardo Costa $^{3}$ and René A. Méndez ${ }^{3}$ \\ ${ }^{1}$ Instituto de Astrofísica de Canarias, Spain \\ ${ }^{2}$ University of Minnesota, Department of Astronomy, USA \\ ${ }^{3}$ Universidad de Chile, Departamento de Astronomía, Chile
}

\begin{abstract}
We present a quantitative analysis of the star formation history (SFH) of 12 fields in the Small Magellanic Cloud (SMC) based on unprecedented deep [(B-R),R] color-magnitude diagrams (CMDs) from Noël et al. (2007). Our fields reach down to the oldest main sequence (MS) turnoff with high photometric accuracy, which is vital for obtaining accurate SFHs. We use the IAC-pop code (Aparicio \& Hidalgo 2009) to obtain the SFH, using a single CMD generated using IAC-star (Aparicio \& Gallart 2004). We find that there are three main periods of enhancement of star formation: a young one peaked at $\sim 0.2-0.5$ Gyr old, only present in the eastern and in the central-most fields; one at intermediate ages, peaked at $\sim 4-5$ Gyr old in all fields; and an old one, peaked at $\sim 10 \mathrm{Gyr}$ in all the fields but the western ones, in which this old enhancement splits into two, peaked at $\sim 8$ Gyr old and at $\sim 12$ Gyr old. This "two-enhancement" zone seems to be a robust feature since it is unaffected when using different stellar evolutionary libraries, implying that stars in the SMC take a Hubble time or more to mix. This indicates that there was a global enhancement in $\psi(t)$ at $\sim 4-5$ Gyr ago in the SMC. We also find that the age of the old population is similar at all radii and at all azimuth and we constrain the age of this oldest population to be older than $\sim 11.5$ Gyr old. The intermediate-age population, in turn, presents variations with both, radii and azimuth. Theoretical studies based on results from larger spatial areas are needed to understand the origin of the young gradient. This young component is highly affected by interactions between Milky Way/LMC/SMC. We do not find yet a region dominated by an old, Milky Way-like, halo at $4.5 \mathrm{kpc}$ from the SMC center, indicating either that this old stellar halo does not exist in the SMC or that its contribution to the stellar populations, at the galactocentric distances of our outermost field, is negligible.
\end{abstract}

Keywords. stars: formation, galaxies: evolution, galaxies: individual (SMC), galaxies: interactions, Magellanic Clouds, galaxies: stellar content, galaxies: structure

\section{Introduction}

Containing stars born over the whole lifetime of a galaxy, the color-magnitude diagram (CMD) is a fossil record of the SFH. For the Milky Way satellites, it is possible to obtain accurate SFHs, from CMDs reaching the oldest main-sequence (MS) turnoffs, using ground-based telescopes. Reaching the oldest MS turnoffs is vital for breaking the age-metallicity degeneracy and properly characterising the intermediate-age and old population (see Gallart et al. 2005). The Magellanic Clouds (MCs), our nearest irregular satellites, provide an ideal environment for this work. In this paper, we will focus on the Small Magellanic Cloud (SMC). The SMC has been historically neglected in favor of its larger neighbor, the Large Magellanic Cloud (LMC). However, there has recently been growing interest in the $\mathrm{SMC}$, related to the discovery from new proper motion measurements - which give constraints to the past orbital motions of the MCs (Kallivayalil et al. 2006; Piatek et al. 2008) - that it may have a different origin to the LMC (see, e.g., Bekki et al. 2004). If true, this would imply that its SFH, evolution and structure could differ significantly from that of the LMC. 


\section{The SFH of the SMC fields}

For each field we obtained three different solutions for the $\mathrm{SFH}, \psi(t, z)$, using three different age binning sets in order to reduce sampling problems associated with binning.

The results for the three different sets were then combined by fitting a cubic spline. The spline fit is the final $\psi(t)$ solution we adopted for each field and, together with the results for the 3 age binning sets, is shown in figure 1. The best-fit SFHs for the binning sets are represented by a different symbol and color in figure 1: red triangles are for age-1, blue squares are for age-2, and green circles are for age-3. Each point carries its vertical error bar that is the formal error from IAC-pop, calculated as the dispersion of 20 solutions with $\chi_{\nu}^{2}=\chi_{\nu, \min }^{2}+1$, where $\chi_{\nu \text {, min }}^{2}$ is that of the solution shown in the figure (see Aparicio \& Hidalgo 2008). Horizontal tracks are not error bars but show the age interval associated to each point. We do not have a constraint on $\psi(t)$ at 13 Gyr old and so the end point of our spline fit is arbitrary. Choosing zero for the end point gives good agreement between the integrated SFH under our spline fit and those of our measured SFHs for the three age binnings.

\subsection{Main characteristics of the $\psi(t)$ solutions for our SMC fields}

As can be seen from figure 1, the eastern fields and the central-most field, smc0057 located in the south - show recent star formation. In particular, the eastern fields show a recent enhancement from $\sim 1.5-2$ Gyr ago until the present, while smc0057 shows a recent peak of star formation $\sim 1$ Gyr ago, which seems to be mostly extinguished at the present time. These $\psi(t)$ peaks are quantified in figure 2 , in which the intensity of each $\psi(t)$ enhancement as a function of radius and age for all the fields are represented together with the pericenter passages of the SMC (see figure caption for details). The intensity of each enhancement is the area under a gaussian function fitted to the elevation in the spline fit. The $\psi(t)$ enhancements at young ages in the Eastern fields and in smc0057, peaked between $\sim 0.2-0.5$ Gyr ago, are not seen in other fields located at similar galactocentric distances. The age of these young peaks is in agreement with the age found in the Magellanic Bridge by Harris (2007) and by other authors in the wing area of the SMC (see, for example, McCumber et al. 2005). The three eastern fields - the only ones presently forming stars - are located in regions of large amounts of $\mathrm{H}$, unlike the rest of our fields, including smc0057. A conspicuous intermediate-age enhancement peakes between $\sim 4$ and $\sim 5$ Gyr old in all fields. In addition, there is a small enhancement at $\sim 2-2.5$ Gyr old in the southern and in the western fields. This enhancement is shifted toward younger ages, at $\sim 1.5-2$ Gyr old in the eastern fields.

Finally, an old-age enhancement occurs at $\sim 10$ Gyr in the eastern and southern fields, which seems to be "split" into two, at $\sim 8$ and $\sim 12$ Gyr old, in the western fields.

\subsection{Global bursts of star formation in the $S M C$}

Phase mixing in a galaxy occurs when stars initially close in space - for example stars formed in a star forming region — spread out over time because they have slightly different energies and angular momenta. Stars are said to be fully phase mixed if there is no memory left that they were born close together. The rate at which stars phase mix depends on the gravitational potential, on the initial proximity of the stars, and on their orbits. As a consequence of the latter, perfectly circular orbits will never mix in radius, while perfectly radial orbits never mix in angle.

The presence of the $\psi(t)$ enhancement at $\sim 4-5$ Gyr old among all the SMC fields, together with the large variations found for ages younger than $\sim 4$ Gyr old, would suggest that the phase mixing time in the SMC is of the order of $\sim 4$ Gyr. However, we find also evidences for spatial variations at older ages: the western fields present two $\psi(t)$ 


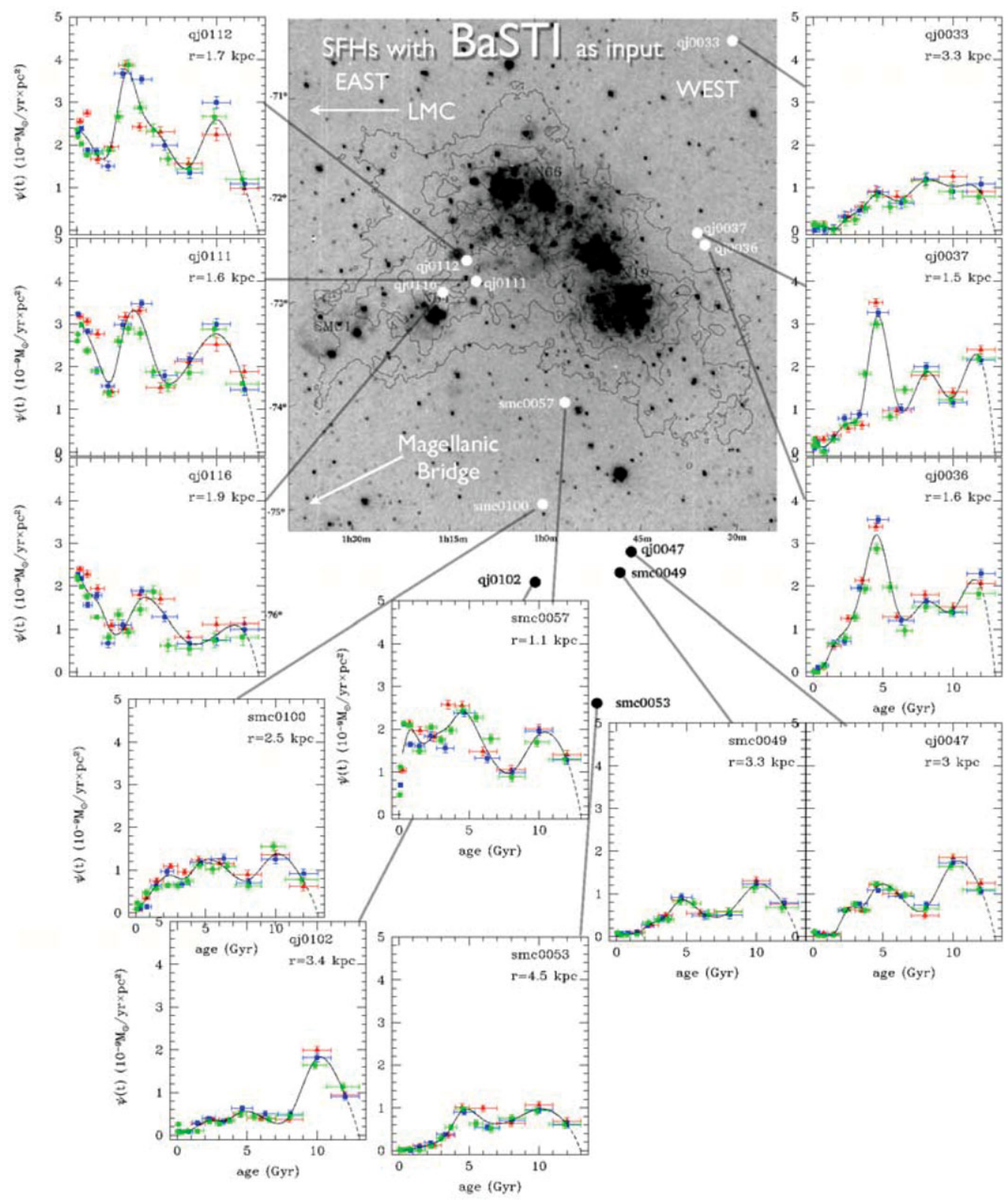

Figure 1. The derived SFHs of our SMC fields. BaSTI stellar evolution library was used as input of IAC-star. Each solution shows the SFH obtained for 3 three age binning schemes (red triangles: age-1, blue squares age-2, and green circles age-3). Each point carries its vertical error bar that is the formal error from IAC-pop, calculated as the dispersion of 20 solutions with $\chi_{\nu}^{2}=\chi_{\nu, \min }^{2}+1$. Horizontal tracks are not error bars, but show the age interval associated to each point. The solid line shows the results of a cubic spline fit to the results. We do not have a constraint on the $\psi(t)$ at 13 Gyr old and the end point of our spline fit was chosen to be zero arbitrarily (dashed lines between 12 and 13 Gyr ago in the spline fit). See text for details. 


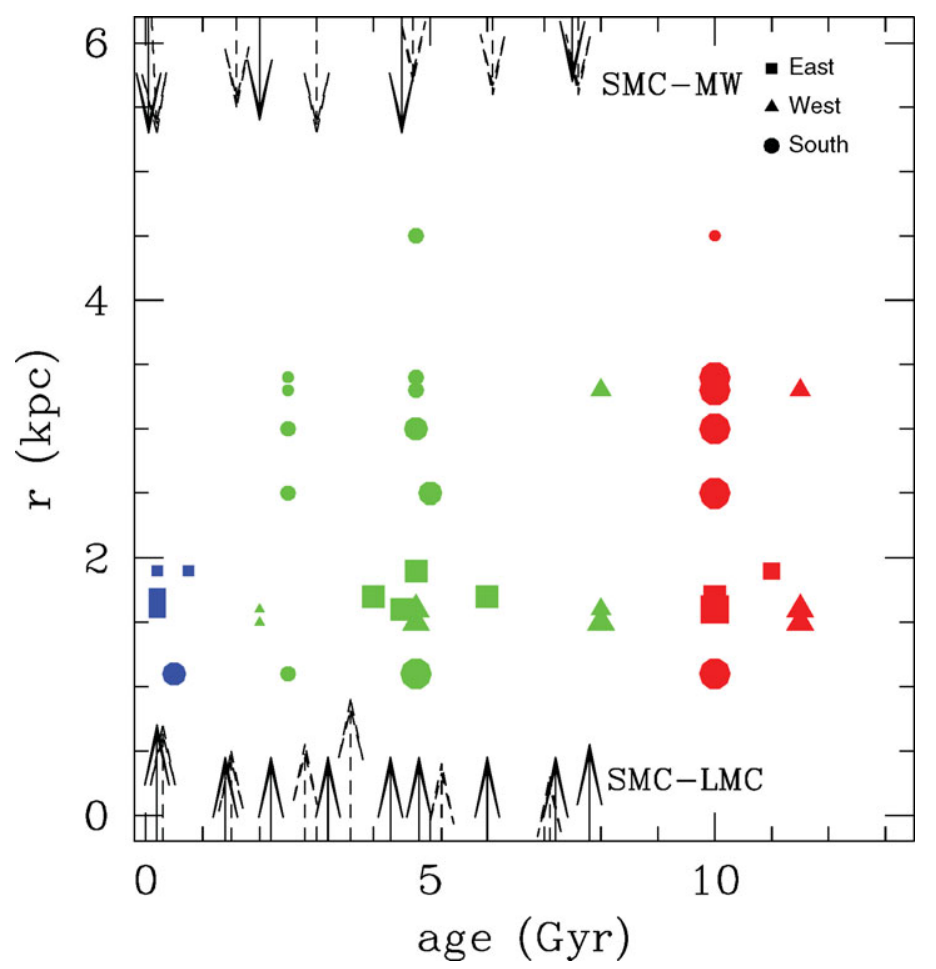

Figure 2. Intensity of the $\psi(t)$ enhancements together with pericenter passages of the SMC. We fitted a gaussian function to the elevations in figure 1 . The size of the symbols depends on the intensity of the enhancement. The bottom arrows indicate the pericenter passages with the LMC while the top arrows show the encounters with the Milky Way (solid-lined arrow represent data from Kallivayalil et al. (2006) and dashed-lined ones are data obtained from Bekki \& Chiba 2005). The size of the arrows represent the intensity of the encounter. Note that some enhancements are hidden behind larger ones. See text for details.

enhancements at $\sim 8$ Gyr old and at $\sim 12$ Gyr old, while in the rest of the fields this old enhancement occurs at $\sim 10$ Gyr old. Time resolution of our SFH solutions is worst for older ages and the results could be model-dependent. However, the spliting of the oldest population in the western fields seems a robust result. On the one hand the two "bumps" remain when sampling the old population with different age binnings. On the other hand, these bumps are impervious when using different stellar evolution libraries. Besides, the formal errors from IAC-pop are significantly smaller than the bumps. In summary, the different behavior of the oldest population in the western fields would imply that stars in the SMC take a Hubble time or more to phase mix. An alternative would be that these variations in older age star distributions might be the result of globular clusters recently dissolved. The following estimative reasoning should be enough to show that this is very unlikely. At least two globulars would be necessary to cause the $\sim 8$ and the $\sim 12$ Gyr old bumps. The mass converted into stars under each bump in each field is of the order of $\sim 2-5 \times 10^{4} \mathrm{M}_{\odot}$. Considering that their traces are extended over a spatial area larger than our three western fields and that not all the stars in the bumps would be expected to originate in the clusters, their initial masses would have been well above $10^{5} \mathrm{M}_{\odot}$, perhaps close to $\sim 10^{6} \mathrm{M}_{\odot}$. Since the evaporation times for such massive clusters are over a Hubble time, the remnants should still be observable, which is not the case. 


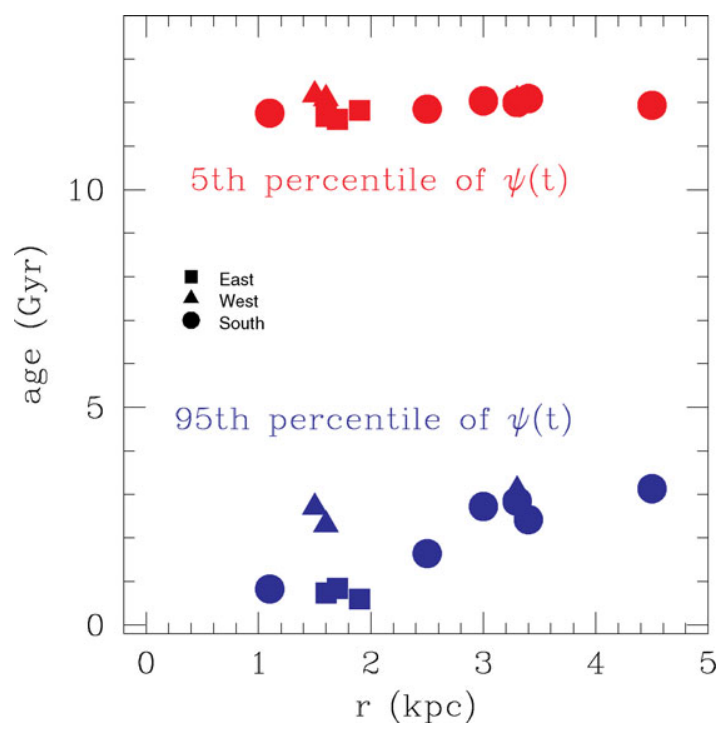

Figure 3. The age at the $5^{\text {th }}$ and at the $95^{\text {th }}$ percentiles of $\psi(t)$ for each of our SMC fields are represented as a function of radius, position angle, and age. See text for details.

We therefore favor the fact that the enhancement of star formation at $\sim 4-5$ Gyr old would be a general burst in the SMC and not a sign of phase mixing over these timescales in this galaxy. However, to confirm our findings more studies are needed with the same depth as the ones presented here but covering larger spatial areas.

\subsection{Spatial distribution of the stellar populations in our SMC fields}

One of the most intriguing issues regarding the SMC evolution is the age and distribution of its oldest stars. In order to shed light into this, we calculated the age at the $5^{\text {th }}$ percentile of $\psi(t)$ in each of our SMC fields, i.e., the population age by which $5 \%$ of the total stars were formed in each field. The results are presented in figure 3 , in which the $5^{\text {th }}$ percentile as a function of radius and age for all the fields is shown. The $5^{\text {th }}$ percentile age in all fields presents a flat distribution at $\sim 11.5$ Gyr. This shows that the age of the old population in all our SMC fields is essentially the same, independently of the galactocentric distance or the position angle. In addition, we constrain the age of the oldest population to be older than $\sim 11.5$ Gyr old.

The asymmetric shape of the SMC main body is known to be exclusively due to its young populations. A way to quantify how this young component is contributing to the irregular appearance, is to find the age at the $95^{\text {th }}$ percentile of $\psi(t)$ in all the SMC fields, which is also presented in figure 3 . The dependence of the $95^{\text {th }}$ percentile age with the position angle is strong: for the southern fields located at $r \gtrsim 3 \mathrm{kpc}$ it occured $\sim 2.5-3$ Gyr ago, at around the same time as in the western fields located at 1.5, 1.6, and $3.3 \mathrm{kpc}$. The three eastern fields and the central-most field, smc0057, are the only ones for which the $95^{\text {th }}$ percentile age is less than 1 Gyr. These facts show that younger star formation episodes did not occur across the entire galaxy at the same time, but rather toward its central southern part and in the wing area.

Another important - and controversial — fact regarding Local Group dwarf galaxies in general and the SMC in particular, is the composition of the outer extended stellar populations. The $95^{\text {th }}$ percentile age for all the SMC fields shows a smooth gradient while 
going further away from the SMC center, indicating that we did not yet reach a region dominated by an old, Milky Way-like, stellar halo at $4.5 \mathrm{kpc}$ from the SMC center. This is stressed by the fact that the $95^{\text {th }}$ percentile age for our outermost field occured at $\sim 3$ Gyr ago. If we would be in such halo-dominated region, the $95^{\text {th }}$ percentile and the $5^{\text {th }}$ percentile age should occur at almost the same time for the outermost fields. Our results are in agreement with Noël \& Gallart (2007) who found that up to $\sim 6.5 \mathrm{kpc}$ from the SMC center, the galaxy is composed by both, intermediate-age and old populations. In summary, our results indicate that either an old, Milky Way-like, stellar halo does not exist in the SMC or that if it exists, its contribution to the stellar population is negligible at $\sim 4.5 \mathrm{kpc}$.

\subsection{Are the $\psi(t)$ enhancements and the $S M C-L M C / S M C-M W$ pericenter passages correlated?}

Several authors, such as Bekki et al. (2004), Bekki \& Chiba (2005) (through numerical simulations) and HZ04 (recovering the SMC SFH) have claimed that the episodes of enhancement in the SFH of the SMC could be related with pericenter passages between the SMC and the LMC and/or between the SMC and the Milky Way. We compare the enhancements in $\psi(t)$ and the pericenter passage times but taking into account the intensity of the $\psi(t)$ enhancements. The intensity of each enhancement is the area under a gaussian function fitted to the elevation in the spline fit. The results are shown in figure 2 . The young enhancements in the eastern fields and in smc0057 between at $\sim 0.2-0.5 \mathrm{Gyr}$ old are not seen in the other fields at the same galactocentric distances. The strongest enhancement at intermediate ages, common to most of the fields (independently of the location in terms of position angle and radius) peaks at $\sim 4.75$ Gyr old. In field qj0112, this enhancement is unfolded into two, peaked at $\sim 4$ Gyr old and at $\sim 6$ Gyr old, respectively. The most conspicuous old enhancement is the one at $\sim 10$ Gyr old which is present in all fields except for the western ones, for which the enhancements at $\sim 8$ and at $\sim 12 \mathrm{Gyr}$ old, mentioned above, are instead very noticeable.

The SMC-LMC and SMC-Milky Way pericenter passages according to Kallivayalil et al. (2006) and Bekki \& Chiba (2005) are shown by the arrows in figure 2. There seems to be a correlation between SMC-Milky Way encounters given by Kallivayalil et al. (2006) and the enhancements in $\psi(t)$ we found at $\sim 2.5$ Gyr ago, at $\sim 4.75$ Gyr ago and at $\sim 8$ Gyr ago.

\section{References}

Aparicio, A. \& Gallart, C. 2004, AJ, 128, 1465

Aparicio, A. \& Hidalgo, S. L. 2009, AJ, submitted

Bekki, K., Couch, W. J., Beasley, M. A., Forbes, D. A., Chiba, M., \& Da Costa, G. S. 2004, ApJ, 610, L93

Bekki, K. \& Chiba, M. 2005, ApJ, 625, L107

Gallart, C., Zoccali, M., \& Aparicio, A. 2005, ARAA, 43, 10

Harris, J. 2007, ApJ 658, 345

Kallivayalil, N., van der Marel, R. P., \& Alcock, C. 2006, ApJ, 652, 1213

McCumber, M. P., Garnett, D. R., \& Dufour, R. J. 2005, AJ, 130, 1083

Noël, N. E. D., Gallart, C., Costa, E., \& Méndez, R. A. 2007, AJ, 133, 2037

Noël, N. E. D. \& Gallart, C. 2007, ApJ, 665, L23

Piatek S., Pryor, C., \& Olszewski, E. W. 2008, AJ, 135, 1024 